\title{
Valor pronóstico de la hiperuricemia en la insuficiencia cardíaca crónica
}

\author{
Alejandro Martínez S, Alejandro González $\mathrm{M}^{\mathrm{a}}$, \\ Cristián Cerda D, Pedro Pérez C, Pablo Castro G, \\ Osvaldo Pérez P, Rodrigo Isa P, Ramón Corbalán H. \\ Prognostic value of hyperuricemia in \\ chronic heart failure
}

Background: Hyperuricemia has been proposed as a risk marker in chronic heart failure, but its value as an independent prognostic is not well established. Aim: To determine the prognostic value of hyperuricemia, in patients with chronic stable heart failure. Patients and methods: Forty six male patients with chronic heart failure, aged $62 \pm 13$ years, were studied. Their ejection fraction was less than $40 \%$ and their serum creatinine was less than $2 \mathrm{mg} / \mathrm{dl}$. Serum uric acid and catecholamines, maximal oxygen consumption $\left(\mathrm{VO}_{2}\right.$ max) and left ventricular ejection fraction were measured. Mortality and the need for cardiac transplant were recorded as endpoints during a mean follow up of $39 \pm 18$ months. The relationship between basal measures and the occurrence of events was analyzed using univariate and multivariate methods. Results: Basal $\mathrm{VO}_{2}$ max and left ventricular ejection fraction were $16 \pm 4.6 \mathrm{ml} / \mathrm{kg} / \mathrm{min}$ and $22 \pm 7 \%$ respectively. Eighteen patients died and three required transplantation during the follow up. Patients reaching these endpoints had a lower $\mathrm{VO}_{2}$ max and left ventricular ejection fraction and higher uric acid levels. Multivariate analysis accepted left ventricular ejection fraction (relative risk $0.89,95 \%$ CI 0.82-0.97) and serum uric acid (relative risk 1.335 95\% CI 1.02-1.74) as significant predictors of events. The relative risk for cardiac transplantation was 7.07 times higher among those with a serum uric acid over $7 \mathrm{mg} / \mathrm{dl}$. Conclusions: A high serum uric acid is an independent predictor of bad prognosis in patients with stable chronic heart failure (Rev Méd Chile 2004; 132: 1031-6).

(Key Words: Heart failure, congestive; Hyperuricemia; Stroke volume; Ventricular ejection fraction)

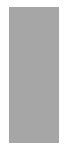

Recibido el 10 de octubre, 2003. Aceptado en versión corregida el 21 de julio, 2004.

Departamento de Enfermedades Cardiovasculares. Hospital Clínico Pontificia Universidad Católica de Chile. Santiago de Chile.

aEstudiante de Medicina

Correspondencia a: Alejandro Martínez S. Fax: 6380227.

Teléfono: 6863218. E mail: amartine@med.puc.cl 
A pesar de avances importantes en su tratamiento, la insuficiencia cardíaca crónica (ICC) continúa siendo una enfermedad progresiva y de alta mortalidad ${ }^{1}$. En ella se asocian trastornos hemodinámicos, neurohumorales y metabólicos, de cuya interacción parece resultar la evolución y el pronóstico de la enfermedad. Entre estos trastornos se han establecido, como factores independientes de mal pronóstico, la severidad del compromiso hemodinámico y el grado de activación neurohumoral. Así, se aceptan como parámetros de alto riesgo, la menor elevación del consumo de oxígeno máximo en ejercicio, el deterioro de la función ventricular izquierda y la elevación de las catecolaminas plasmáticas en reposo y esfuerzo ${ }^{2}$. Sólo en los últimos años se estudia la importancia pronóstica de los trastornos metabólicos. Entre éstos, ha adquirido alto interés el estudio de las alteraciones del metabolismo del ácido úrico.

Los niveles plasmáticos de ácido úrico frecuentemente se encuentran elevados en la ICC. Tradicionalmente esta elevación se ha atribuido a una disminución en la excreción secundaria al uso de diuréticos y al defecto de la perfusión renal, y no se le ha dado un rol en la evolución de la enfermedad. Recientemente, sin embargo, Anker y cols $^{3}$ comunicaron que los niveles plasmáticos elevados de ácido úrico, independiente de la función renal y del uso de diuréticos, predicen mejor que otros parámetros el riesgo de mortalidad y necesidad de trasplante en pacientes con ICC. Este hallazgo no sólo tiene un valor potencial en la estimación del pronóstico, sino que también sugiere que en la ICC puede haber una mayor producción de ácido úrico por un mecanismo eventualmente ligado a la progresión de la enfermedad. Anker y cols sugieren que la elevación del ácido úrico traduciría una mayor activación de la xantinooxidasa, enzima que podría afectar la evolución de la ICC a través de la producción de estrés oxidativo, ya que es uno de los sistemas enzimáticos más importantes en la generación de radicales libres. Sin embargo, llama la atención el elevado nivel de corte que ellos encontraron como mejor predictor de mortalidad $(9,8 \mathrm{mg} / \mathrm{dL})$. Estos niveles los hemos observado sólo en casos muy extremos de ICC y pueden estar asociados a defectos acentuados de perfusión renal y sistémicos, los que producen aumento de la síntesis de ácido úrico por hipoxia celular y disminución de su excreción renal ${ }^{4}$. Por lo tanto, en ese estudio, la hiperuricemia puede ser un reflejo de una enfermedad más avanzada y no necesariamente su asociación con mortalidad se puede atribuir a que el mecanismo xantinooxidasa sea importante en el pronóstico.

Por este motivo hemos evaluado en pacientes ambulatorios con ICC de severidad moderada y estables, el valor pronóstico de la hiperuricemia, en relación al riesgo de mortalidad o trasplante cardíaco, en comparación con otros factores que conocidamente se asocian a mayor riesgo en la ICC, como son el consumo máximo de oxígeno $\left(\mathrm{VO}_{2}\right.$ máx), la fracción de eyección del ventrículo izquierdo (FEVI) y la determinación de noradrenalina plasmática (NAD).

\section{MATERIAL Y MÉTODOS}

Pacientes. La población se seleccionó de nuestra base de datos de pacientes ambulatorios con ICC estable, estudiados con la determinación de uricemia, creatininemia, $\mathrm{VO}_{2}$ máx, FEVI y $\mathrm{NAD}$, que fueron seguidos por un período mayor a un año. Se incluyó sólo a los hombres, con FEVI menor de $40 \%$, creatinina plasmática menor de $2 \mathrm{mg} / \mathrm{dL}$, sin antecedentes de gota o uso de allopurinol y que no recibían más de dos comprimidos diarios de furosemida o hidroclorotiazida. Las mujeres no fueron incluidas, porque representan una proporción menor en esta población y como género tienen niveles de ácido úrico inferiores, por lo que requerirían un análisis separado.

Mediciones basales. Se realizaron en forma ambulatoria exigiendo que los pacientes estuvieran en tratamiento médico optimizado con digital, diuréticos e inhibidores de la enzima convertidora y en condición clínica estable durante las 4 semanas previas a las evaluaciones. Se determinó el $\mathrm{VO}_{2}$ máx mediante una prueba de esfuerzo (Naughton) con recolección de gases espirados en un equipo Quinton Q-Plex. La FEVI mediante ventriculografía radioisotópica. Los niveles de NAD en sangre venosa periférica se midieron mediante HPLC y los de ácido úrico y creatininemia mediante técnicas estándar de laboratorio. 
Seguimiento. Se efectúo un seguimiento telefónico consignando los eventos de mortalidad por cualquier causa o trasplante cardíaco y el momento de ocurrencia del evento adverso a partir de la fecha de la evaluación basal.

Estadística. Los resultados de las mediciones basales se expresan como promedios \pm desviación estándar y se compararon entre los que presentaron eventos (mortalidad o trasplante) versus los que no los presentaron, mediante análisis univariado, empleando la prueba de $t$ de Student para muestras no pareadas y multivariado a través de la técnica de regresión de Cox. En este análisis se incluyeron paso a paso los valores basales, como variables continuas, de $\mathrm{VO}_{2}$ máx, FEVI, NAD, uricemia y creatininemia, considerando como punto final la ocurrencia de cualquiera de los dos eventos estudiados. El mismo análisis se hizo incluyendo las variables en forma dicotomizada, eligiendo el corte de acuerdo a los niveles conocidos de riesgo para $\mathrm{VO}_{2}$ ( $\leq 15 \mathrm{vs}>15 \mathrm{ml} / \mathrm{kg} / \mathrm{min}$ ), FEVI ( $\leq 28$ vs $>28 \%$ ) y NAD ( $>400$ vs $\leq 400 \mathrm{pg} / \mathrm{dl}$ ), y separando los pacientes con uricemia elevada $(>7$ $\mathrm{mg} / \mathrm{dl})$ vs normal $(\leq 7 \mathrm{mg} / \mathrm{dl})$. Además, se construyeron curvas de sobrevida libre de trasplante usando la técnica de Kaplan-Meier, comparando los pacientes que tenían ácido úrico normal versus los que lo tenían elevado. El análisis estadístico se realizó con el programa SPSS.

\section{RESULTADOS}

Se estudiaron 46 pacientes de $62 \pm 13$ años, con ICC cuya etiología era idiopática en 23 , isquémica en 19 y otras en 4 pacientes. La capacidad funcional en el momento de la evaluación basal era II-III, el consumo máximo de oxígeno promedio de 16,6 64 ,6 $\mathrm{ml} / \mathrm{kg} / \mathrm{min}$ y la fracción de eyección del ventrículo izquierdo de $22 \pm 7 \%$. A todos estos pacientes se les aconsejó mantener el tratamiento médico y fueron seguidos por un promedio de $39 \pm 18$ meses.

Durante el período de seguimiento se consignó mortalidad en 18 pacientes (39\%) y trasplante en $3(6,5 \%)$. Entre los fallecidos, 6 pacientes tenían etiología idiopática, 10 isquémica y los otros 2 tenían valvulopatías operadas. Los 3 pacientes que se sometieron a trasplante cardíaco tenían miocardiopatía idiopática.

En la Tabla 1 se comparan, en un análisis univariado, las variables estudiadas entre los 21 pacientes que presentaron eventos versus los 25 que no los presentaron. Resultaron significativas las diferencias entre $\mathrm{VO}_{2}$ máx $(15,1 \pm 4,6$ vs $17,7 \pm 4,3 ; p=0,01)$, FEVI $(18 \pm 7$ vs $24 \pm 7 ; p=0,003)$ y uricemia $(8,2 \pm 2,4$ vs $6,9 \pm 1,9 ; p=0,01)$.

En el análisis multivariado, incluyendo los valores de las mediciones como variables continuas, sólo la reducción de la FEVI ( $\mathrm{p}=0,009$; $\mathrm{RR}=0,895 ; 95 \% \mathrm{IC}=0,82-0,97)$ y la elevación de la

Tabla 1. Comparación de las características basales de los pacientes que presentaron eventos de mortalidad o trasplante versus los que no tuvieron estos eventos. Análisis univariado. Test de la t de Student no pareado

\begin{tabular}{|lccc|}
\hline & $\begin{array}{c}\text { Mortalidad } 0 \\
\text { trasplante } \\
\mathrm{n}=21\end{array}$ & Sin eventos & $\mathrm{p}$ \\
& $\mathrm{n}=25$ & \\
Edad (años) & $60 \pm 15$ & $63 \pm 12$ & $\mathrm{NS}$ \\
$\mathrm{VO}$ máx (ml/kg/min) & $15,1 \pm 4,6$ & $17,7 \pm 4,3$ & 0,01 \\
$\mathrm{FEVI}(\%)$ & $18 \pm 7$ & $24 \pm 7$ & 0,003 \\
$\mathrm{NAD}(\mathrm{pg} / \mathrm{ml})$ & $707 \pm 398$ & $572 \pm 263$ & $\mathrm{NS}$ \\
Uricemia (mg/dl) & $8,2 \pm 2,4$ & $6,9 \pm 1,9$ & 0,01 \\
Creatininemia (mg/dl) & $1,26 \pm 0,19$ & $1,26 \pm 0,28$ & $\mathrm{NS}$ \\
\hline
\end{tabular}

$\mathrm{VO}_{2}$ máx: Consumo de oxígeno máximo. FEVI: fracción de eyección del ventrículo izquierdo. NAD: noradrenalina plasmática. 
Tabla 2. Riesgo relativo de mortalidad o trasplante de acuerdo a las mediciones basales. Análisis de regresión multivariado de C ox

\begin{tabular}{|lcc|}
\hline & $\mathrm{RR}(95 \% \mathrm{IC})$ & $\mathrm{p}$ \\
\hline FEVI $(\leq 28$ vs $>28 \%)$ & $4,64(1,09-19,74)$ & 0,037 \\
$\mathrm{VO}_{2}$ máx $(\leq 15$ vs $>15 \mathrm{ml} / \mathrm{kg} / \mathrm{min})$ & $4,03(1,36-11,96)$ & 0,012 \\
Uricemia $(>7$ vs $\leq 7 \mathrm{mg} / \mathrm{dl})$ & $7,07(1,94-25,8)$ & 0,003 \\
\hline
\end{tabular}

FEVI: fracción de eyección del ventrículo izquierdo. $\mathrm{VO}_{2}$ máx: Consumo de oxígeno máximo.

uricemia $(\mathrm{p}=0,03 ; \mathrm{RR}=1,335 ; 95 \% \mathrm{IC}=1,02-1,74)$, predijeron en forma independiente el riesgo de mortalidad y trasplante.

Considerando las variables dicotomizadas, con los niveles de corte preestablecidos, se agrega como determinante de riesgo el $\mathrm{VO}_{2} \leq 15 \mathrm{ml} / \mathrm{kg} /$ min y se confirma el mayor riesgo relativo de la reducción de la FEVI y de la hiperuricemia (Tabla 2). El riesgo relativo de mortalidad o trasplante fue de 7,07 veces en los que tenían hiperuricemia ( $>7$ $\mathrm{mg} \%$ ) comparados con los que tenían uricemia normal ( $\leq 7 \mathrm{mg} \%)$, lo que alcanza alta significación estadística $(p=0,003)$. En la Figura 1 se compara las curvas de Kaplan-Meir de sobrevida sin tras- plante entre los pacientes con uricemia normal ( $\leq 7 \mathrm{mg} / \mathrm{dL})$ y los que tenían hiperuricemia ( $>7$ $\mathrm{mg} / \mathrm{dL}$ ), demostrándose una amplia diferencia que favorece a los que tenían uricemia normal.

Aunque los pacientes con hiperuricemia tenían sus niveles de creatinina plasmática levemente superiores $(1,32 \pm 0,25$ vs $1,19 \pm 0,23 ; p=0,04)$, los datos no apoyan que este factor genere la diferencia. La creatininemia basal no fue diferente entre los pacientes que presentaron eventos comparados con los que no los tuvieron $(1,26 \pm 0,19 \mathrm{mg} / \mathrm{dl}$ vs $1,26 \pm 0,28 \mathrm{mg} / \mathrm{dl}$ ) y en el análisis multivariado el valor pronóstico de la hiperuricemia persiste con o sin la inclusión de la creatininemia.

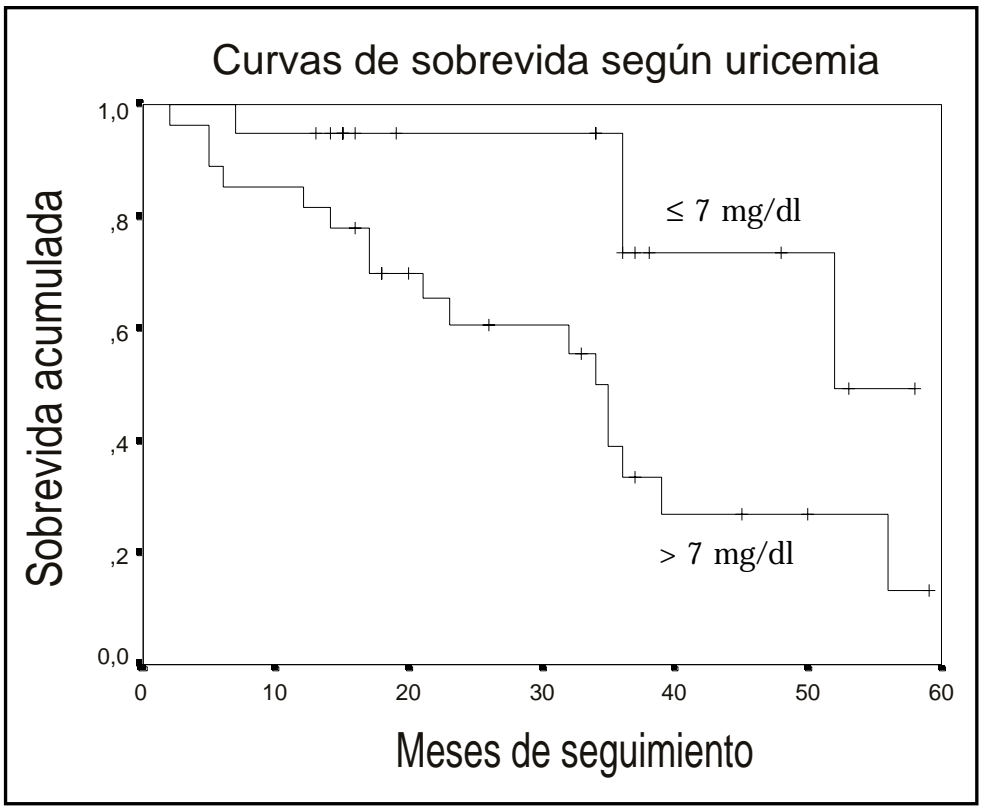

Figura 1. Curvas de sobrevida de Kaplan-Meier entre los pacientes con uricemia normal $(\leq 7 \mathrm{mg} / \mathrm{dl})$ y los pacientes con uricemia elevada $(>7 \mathrm{mg} / \mathrm{dl}), \mathrm{p}=0,008$. 


\section{DisCUSIÓN}

Los resultados de este estudio revelan que la hiperuricemia es un factor de mal pronóstico independiente de creatininemia, $\mathrm{VO}_{2}$ máx, FEVI y NAD, en pacientes con ICC sin insuficiencia renal. En estos pacientes, la elevación de la uricemia por encima de $7 \mathrm{mg} / \mathrm{dL}$ determina una curva de sobrevida significativamente peor. Este hallazgo, comunicado previamente por Anker y cols en pacientes con ICC avanzada ${ }^{3}$, con nuestros datos se extiende a pacientes con ICC moderada.

El hecho de que en nuestro estudio este resultado se haya obtenido en pacientes sin insuficiencia renal o uso de dosis altas de diuréticos, e independiente de la creatininemia, plantea la posibilidad de que la elevación de ácido úrico está asociada a un aumento de su producción y eventualmente vinculado a un mecanismo involucrado con la progresión de la enfermedad.

Desde un punto de vista teórico el aumento de la producción de ácido úrico en la ICC podría deberse a un aumento de la actividad de la xantinooxidasa ${ }^{5,6}$ y también a una mayor disponibilidad del sustrato para la enzima, como ocurre durante la hipoxia celular. En este último caso, la reducción del aporte de oxígeno a las células determina que el consumo de ATP, sin su adecuada regeneración, favorece la degradación de nucleótidos de adenina a inosina, hipoxantina, xantina y ácido úrico ${ }^{6}$. En nuestros pacientes con ICC moderada es poco probable un defecto severo de la oxigenación celular, por lo tanto, suponemos que el factor más importante de la elevación del ácido úrico debe estar relacionado a un aumento de la actividad de la xantinooxidasa.

Nosotros no medimos la xantinooxidasa, pero en otros estudios se ha encontrado que en pacien-

\section{REFERENCIAS}

1. Macintyre K, Capewell S, Stewart S, Chalmers J, Boyd J, Finlayson A et aL. Evidence of improving prognosis in heart failure: trends in case fatality in 66.547 patients hospitalized between 1986 and 1995. Circulation 2000; 102: 1126-31.

2. Cohn JN, Johnson GR, Shabetai R, Loeb H, Tristani F, RECTOR T ET AL. Ejection fraction, peak exercise tes con ICC su actividad se encuentra elevada tanto en el plasma ${ }^{7}$, como unido al endotelio de los vasos periféricos ${ }^{7}$ y también en el miocardio ${ }^{5}$. Este incremento se ha explicado por diferentes mecanismos. Puede ocurrir por estímulo de citoquinas, que aumentan la expresión del gen de la xantinodehidrogenasa ${ }^{8}$, también bajo el estímulo de la hipoxia celular que determina aumento de sustrato ${ }^{9}$ y probablemente por la activación del sistema renina-angiotensina, porque se ha visto que la inhibición de la xantinooxidasa puede mejorar significativamente la vasodilatación dependiente de endotelio en ratas con sobreexpresión del sistema renina-angiotensina ${ }^{10}$.

La elevación de la xantinooxidasa podría explicar el valor pronóstico de la hiperuricemia en la ICC porque esta enzima es uno de los mecanismos enzimáticos reconocidos como fuente de radicales libres en la $\mathrm{ICC}^{11}$. Al mismo tiempo que la xantinooxidasa cataliza dos reacciones que permiten la producción de ácido úrico, genera radicales libres de oxígeno ${ }^{6}$. Los radicales libres y el estrés oxidativo, a su vez, se han vinculado tanto con los fenómenos asociados al remodelamiento ventricular ${ }^{12}$ como a los trastornos de la circulación periférica ${ }^{7}$ que se encuentran en estos pacientes. Al mismo tiempo hay datos que sugieren que la inhibición de la xantinooxidasa con allopurinol puede mejorar tanto la función cardía$\mathrm{ca}^{13}$ como la función endotelial ${ }^{14,15}$.

En conclusión, nuestros hallazgos confirman que la elevación del ácido úrico tiene un valor pronóstico en los pacientes con ICC estable y sugieren fuertemente que una alteración en su mecanismo de producción puede jugar un rol en la evolución de la enfermedad. Para confirmar esta hipótesis se requiere un estudio de intervención sobre el metabolismo del ácido úrico.

oxygen consumption, cardiothoracic ratio, ventricular arrhymias, and plasma norepinephrine as determinants of prognosis in heart failure. Circulation 1993; 87: VI5-16.

3. AnKer S, Doehner W, Rauchaus M, Sharma R, Francis D, Knosalia C et al. Uric acid and survival in chronic heart failure: validation and application in metabolic, functional and hemodynamic staging. Circulation 2003; 107: 1991-7. 
4. Martínez A, Pérez P, Ossa C, Corbalán R, Jalil J, CASTRO P ET AL. La hiperuricemia es un marcador del umbral anaeróbico en la insuficiencia cardíaca crónica. Rev Méd Chile 2001; 129: 499-504.

5. De Jong JW, Schoemaker RG, De Jonge R, Bernocchi P, Keijzer E, Harrison R et al. Enhanced expression and activity of xanthine oxidoreductase in the failing heart. J Mol Cell Cardiol 2000; 32: 2083-9.

6. Sawyer D, Siwik D, Xiao L, Pimentel D, Singh K, Colucci W. Role of Oxidative Stress in Myocardial Hypertrophy and Failure. J Mol Cell Cardiol 2002; 34: 379-88.

7. Landmesser U, Spiekermann S, Dikalov S, Tatge H, WiLKe R, KoHLer C et al. Vascular oxidative stress and endothelial dysfunction in patients with chronic heart failure. Role of xanthine-oxidase and extracellular superoxide dismutase. Circulation 2002; 106: 3073-8.

8. Dupont GP, Huecksteadt TP, Marshall BC, Ryan US, Michael JR, Hoidal JR. Regulation of xanthine dehydrogenase and xanthine oxidase activity and gene expression in cultured rat pulmonary endothelial cells. J Clin Invest 1992; 89: 197-202.

9. Hassoun PM, Yu FS, Shedd AL, Zulueta JJ, ThannicKaL VJ, LanZILO JJ ET AL. Regulation of endothelial xanthine dehydrogenase/xanthine oxidase gene expression by oxygen tension. Am J Physiol 1994; 266: L163-71.

10. MervaAla EM, Cheng ZJ, Tikkanen I, Lapatto R, NuRminen K, VapaAtalo H et al. Endothelial dysfunction and xanthine oxidoreductase activity in rats with human renin and angiotensinogen genes. Hypertension 2001; 37: 414-8.

11. DhaLa NS, Temsah RM, Netticadan T. Role of oxidative stress in cardiovascular diseases. J Hypertens 2000; 18: 655-73.

12. Ide $\mathrm{T}$, Tsutsui $\mathrm{H}$, Kinugawa S, Suematsu N, HayashiDANI S, IChIKaWA $\mathrm{K}$ et aL. Direct evidence for increased hydroxyl radicals originating from superoxide in the failing human myocardium. Circ Res 2000; 86: 152-7.

13. Cappola tP, Kass DA, Nelson GS, Berger RD, Rosas GO, KobeISSI ZA ET AL. Allopurinol improves myocardial efficiency in patients with idiopathic dilated cardiomyopathy. Circulation 2001; 104: 2407-11.

14. Doehner W, Schoene N, Rauchhau S, Leyva-Leon F, Pavitt D, Reaveley D et al. Effects of xanthine oxidase inhibition with allopurinol on endothelial function and peripheral blood flow in hyperuricemic patients with chronic heart failure. Results from 2 placebo-controlled studies. Circulation 2002; 105: 2619-24.

15. Farquharson CA, Butler R, Hill A, Belch JJ, STRUTHERS AD. Allopurinol improves endothelial dysfunction in chronic heart failure. Circulation 2002; 106: 221-6.

Agradecimientos

Se agradece al Dr. Gastón Chamorro S., por su valiosa colaboración en la revisión del manuscrito. 